\title{
Coexistence of ordinary gyration and weak optical activity in crystals: eigen waves of a new type
}

\author{
Zapeka B., Kostyrko M. and Vlokh R. \\ Institute of Physical Optics, 23 Dragomanov St., 79005 Lviv, Ukraine \\ E-mail: vlokh@ifo.lviv.ua
}

Received: 07.05.2010

\begin{abstract}
We present the results of analysis for the refractive indices and the ellipticity of eigen waves under the conditions of coexisting gyration and weak optical activity. Quantitative relations describing the appropriate parameters are derived. It is shown that the ellipticities of eigen waves become different and one of that waves acquires a complicated (longitudinally-transverse) elliptical polarisation state. This represents a new type of electromagnetic eigen waves in crystals.
\end{abstract}

Keywords: weak optical activity, gyration, spatial dispersion, refraction, polarisation

PACS: $78.20 . \mathrm{Ek}$

UDC: 535.56

\section{Introduction}

Optical activity is described with taking into consideration an inhomogeneity of the electric displacement $D_{j}$ of an optical wave propagating through a medium:

$$
E_{i}=B_{i j}^{0} D_{j}+i \gamma_{i j k} \frac{\partial D_{j}}{\partial x_{k}}
$$

where $E_{i}$ denotes electric field, $B_{i j}^{0}$ the optical-frequency impermeability tensor without accounting for spatial dispersion effects, $\gamma_{i j k}$ the third-rank antisymmetric polar tensor $\left(\gamma_{i j k}=-\gamma_{j i k}\right)$, and $x_{k}$ the coordinate. Using the known duality relation,

$$
\frac{2 \pi}{\lambda} \gamma_{i j k}=\delta_{i j l} g_{l k}
$$

one can write the tensor $\gamma_{i j k}$ as an axial asymmetric second-rank gyration tensor $g_{l k}$, with $\delta_{i j l}$ being the unit antisymmetric Levi-Civita tensor and $\lambda$ the wavelength of light. Then simple transformations valid for a plane electromagnetic wave, yield in

$$
E_{i}=\left(B_{i j}^{0}+i \delta_{i j l} g_{l k} m_{k}\right) D_{j}
$$

where $k_{k}=\frac{2 \pi}{\lambda} m_{k}$ is the wave vector of light and $m_{k}$ the unit vector parallel to $k_{k}$. The asymmetric gyration tensor can be decomposed into its symmetric and antisymmetric 
parts:

$$
g_{l k}=g_{l k}^{s}+g_{l k}^{a s},
$$

where the antisymmetric part is dual to some polar vector $h_{r}$,

$$
g_{l k}^{a s}=\delta_{l k r} h_{r}
$$

and describes a so-called weak optical activity [1-6], while the symmetric part takes account of a usual optical activity often called as a gyration.

In our recent study [7] we have shown that the refractive indices $n_{1}, n_{2}$, the optical birefringence $\Delta n_{32}$ and the ellipticity of the eigen waves $\kappa$ in the presence of the pure weak optical activity are described by the following relations:

$$
\begin{aligned}
& n_{3}=n_{03}-\frac{1}{2} n_{03}^{3}\left[h_{3} \times m_{1}\right]^{2}=n_{03}-\frac{1}{2} n_{03}^{3} h_{3}^{2}, \\
& \Delta n_{32}=\Delta n_{32}^{0}-\frac{1}{2} n_{03}^{3}\left[h_{3} \times m_{1}\right]^{2}=\Delta n_{32}^{0}-\frac{1}{2} n_{03}^{3} h_{3}^{2}, \\
& \kappa=\frac{\bar{n}^{3} h_{3}^{2}}{2 \Delta n_{23}^{0}},
\end{aligned}
$$

where $n_{02}, n_{03}$ and $\Delta n_{32}^{0}=n_{03}-n_{02}$ are respectively the refractive indices and the birefringence for the case if the spatial dispersion is disregarded, and $\bar{n}=\left(n_{02}+n_{03}\right) / 2$ is the mean refractive index.

The weak optical activity manifests itself as a single crystal optical effect, i.e. without the accompanying ordinary gyration, in the crystals or other anisotropic media which belong to the point symmetry groups $3 \mathrm{~m}, 4 \mathrm{~mm}, 6 \mathrm{~mm}$, and $\infty \mathrm{mm}$. On the contrary, the weak optical activity is forbidden by the symmetry in the gyrotropic crystals which have the symmetry $222,32,422,432, \overline{4} 2 m$, and $\overline{4}$. At the same time, the both effects coexist in the gyrotropic crystals of the point symmetries $1,2, m m 2, m, 3,4$, and 6 . Thus, a reasonable question appears in the latter case: how does the coexistence of the ordinary and weak optical activities affect the character of electromagnetic eigen waves and their refractive indices? We would like to remind in this respect that, in the presence of the weak optical activity only, an initially linearly polarised wave with its polarisation parallel to the higher-fold symmetry axis (in particular, the optic axis, i.e. $Z$ axis) is transformed into a longitudinal elliptically polarised one after passing through the crystal. In other words, the electric field component $E_{1}$ of the wave becomes nonzero when the light propagates along the $X$ direction. The orthogonal eigen wave, which oscillates parallel to the $Y$ axis, remains linearly polarised at the same conditions.

The present work is devoted to analysis of the eigen waves and their refractive indices for the most general case when the weak optical activity and the ordinary gyration are superimposed. 


\section{Refractive indices and the ellipticity of eigen waves}

In the presence of both the weak optical activity and the ordinary gyration Eq. (3) may be written as

$$
\begin{aligned}
& E_{i}=\left(B_{i j}^{0}+i \delta_{i j l} g_{l k}^{s} m_{k}+i \delta_{i j l} g_{l k}^{a s} m_{k}\right) D_{j}=\left(B_{i j}^{0}+i \delta_{i j l} g_{l k}^{s} m_{k}+i \delta_{i j l} \delta_{l k r}\left[h_{r} \times m_{k}\right]\right) D_{j} \\
& =\left(B_{i j}^{0}+i \Delta_{1}+i \Delta_{2}\right) D_{j} .
\end{aligned}
$$

To begin with, let us consider an anisotropic optically uniaxial crystal in which only ordinary gyration exists $\left(\Delta_{1}=\delta_{i j l} g_{l k}^{s} m_{k}=g_{11} \neq 0, \quad \Delta_{2}=\delta_{i j l} \delta_{l k r}\left[h_{r} \times m_{k}\right]=h_{3}=0 \quad\right.$ and $B_{11}^{0}=B_{22}^{0} \neq B_{33}^{0}$ ). Let the optic axis be parallel to the $Z$ axis and the optical wave propagate along the $X$ direction $\left(m_{2}=m_{3}=0, m_{1}=1\right)$. The matrix that couples the components of the electric field and the electric displacement is given by

$$
\begin{array}{c|ccc} 
& D_{1} & D_{2} & D_{3} \\
\hline E_{1} & B_{11}^{0} & 0 & 0 \\
E_{2} & 0 & B_{11}^{0} & i \Delta_{1} \\
E_{3} & 0 & -i \Delta_{1} & B_{33}^{0}
\end{array},
$$

so that we get the following system of equations:

$$
\left\{\begin{array}{l}
E_{2}=B_{11}^{0} D_{2}+i \Delta_{1} D_{3}, \\
E_{3}=-i \Delta_{1} D_{2}+B_{33}^{0} D_{3} .
\end{array}\right.
$$

The impermeability tensor components and the refractive indices with taking of the gyration into account are equal to

$$
\begin{aligned}
& B_{11}^{n}=B_{11}^{0}, \\
& B_{22}^{n}=\left(\frac{1}{n_{2}^{2}}\right)^{n}=\frac{1}{n_{02}^{2}}-\frac{\Delta_{1}^{2}}{\frac{1}{n_{03}^{2}}-\frac{1}{n_{02}^{2}}} \simeq \frac{1}{n_{02}^{2}}+\frac{\bar{n}^{3} g_{11}^{2}}{2 \Delta n^{0}}, \\
& B_{33}^{n}=\left(\frac{1}{n_{3}^{2}}\right)^{n}=\frac{1}{n_{03}^{2}}+\frac{\Delta_{1}^{2}}{\frac{1}{n_{03}^{2}}-\frac{1}{n_{02}^{2}}} \simeq \frac{1}{n_{03}^{2}}-\frac{\bar{n}^{3} g_{11}^{2}}{2 \Delta n^{0}} \\
& n_{1}^{n}=n_{01}, \\
& n_{2}^{n}=n_{02}-\frac{\bar{n}^{6} g_{11}^{2}}{4 \Delta n^{0}} \\
& n_{3}^{n}=n_{03}+\frac{\bar{n}^{6} g_{11}^{2}}{4 \Delta n^{0}}
\end{aligned}
$$

where $\bar{n}$ and $\Delta n^{0}$ imply the average values of the refractive indices and the initial birefringence, respectively. As seen from Eqs. (10) and (11), the account of the gyration leads to changes in the impermeability components and the refractive indices, i.e. 
$B_{22}^{n} \neq B_{11}^{n}, n_{1}^{n} \neq n_{2}^{n}$. Notice that the above equations coincide with those presented, e.g., in the monograph [8] for description of the refractive indices of anisotropic media modified by the gyration. Hence, the ellipticities for the linearly polarised incident wave parallel to the $Y$ and $Z$ axes are determined by the relations

$$
\begin{aligned}
& \frac{D_{3}^{(2)}}{D_{2}^{(2)}}=-i \kappa=-i \frac{\bar{n}^{3} g_{11}}{2 \Delta n^{0}}, \quad \kappa=-\frac{\bar{n}^{3} g_{11}}{2 \Delta n^{0}}, \\
& \frac{D_{2}^{(3)}}{D_{3}^{(3)}}=i \kappa=i \frac{\bar{n}^{3} g_{11}}{2 \Delta n^{0}}, \quad \kappa=\frac{\bar{n}^{3} g_{11}}{2 \Delta n^{0}},
\end{aligned}
$$

respectively. The ellipse of polarisation in case of the pure ordinary gyration effect lies in the $Z Y$ plane.

Considering the relations derived above, in the presence of the weak optical activity $\left(\Delta_{2} \neq 0\right)$ we arrive at the matrix

\begin{tabular}{c|cc} 
& $D_{1}$ & $D_{3}$ \\
\hline$E_{1}$ & $B_{11}^{0}$ & $i \Delta_{2}$ \\
$E_{3}$ & $-i \Delta_{2}$ & $B_{33}^{n}$
\end{tabular}

and the system of equations

$$
\left\{\begin{array}{l}
E_{1}=B_{11}^{0} D_{1}+i \Delta_{2} D_{3}, \\
E_{3}=-i \Delta_{2} D_{1}+B_{33}^{n} D_{3} .
\end{array}\right.
$$

Since $B_{33}^{n}=n_{03}^{-2}-\bar{n}^{3} \Delta_{1}^{2} / 2 \Delta n$, the impermeability tensor components modified by the mutual influence of both the ordinary gyration and the weak optical activity are found to be

$$
\begin{aligned}
& B_{11}=\left(\frac{1}{n_{1}^{2}}\right)=\frac{1}{n_{01}^{2}}+\frac{2 \bar{n}^{3} \Delta n^{0} \Delta_{2}^{2}}{4 \Delta n^{02}-\bar{n}^{6} \Delta_{1}^{2}}, \quad B_{22}=\left(\frac{1}{n_{2}^{2}}\right)=\frac{1}{n_{02}^{2}}-\frac{\bar{n}^{3} \Delta_{1}^{2}}{2 \Delta n^{o}}, \\
& B_{33}=\left(\frac{1}{n_{3}^{2}}\right)=\frac{1}{n_{03}^{2}}+\frac{\bar{n}^{3}}{2 \Delta n^{o}} \Delta_{1}^{2}-\frac{2 \bar{n}^{3} \Delta n^{0} \Delta_{2}^{2}}{4 \Delta n^{02}-\bar{n}^{6} \Delta_{1}^{2}} .
\end{aligned}
$$

We mention that these relations are reduced to Eqs. (10) at $\Delta_{2}=0$, while at $\Delta_{1}=0$ one readily obtains the formulae obtained in the work [7]. Let us now take into account that $4 \Delta n^{02} \gg \bar{n}^{6} \Delta_{1}^{2}$ and make use of the fact that $\Delta_{1}=g_{11}$ and $\Delta_{2}=h_{3}$ in our geometry. Then Eqs. (15) may be simplified, resulting in

$$
\begin{aligned}
& B_{11}=\left(\frac{1}{n_{1}^{2}}\right)=\frac{1}{n_{01}^{2}}+\frac{\bar{n}^{3}}{2 \Delta n^{0}} \Delta_{2}^{2}=\frac{1}{n_{01}^{2}}+\frac{\bar{n}^{3}}{2 \Delta n^{0}} h_{3}^{2}, \quad B_{22}=\left(\frac{1}{n_{2}^{2}}\right)=\frac{1}{n_{02}^{2}}-\frac{\bar{n}^{3}}{2 \Delta n^{o}} g_{11}^{2}, \\
& B_{33}=\left(\frac{1}{n_{3}^{2}}\right)=\frac{1}{n_{03}^{2}}+\frac{\bar{n}^{3}}{2 \Delta n^{0}}\left(g_{11}^{2}-h_{3}^{2}\right) .
\end{aligned}
$$

The corresponding refractive indices become as follows: 


$$
\begin{aligned}
& n_{1}=n_{01}-\frac{\bar{n}^{6}}{4 \Delta n^{0}} h_{3}^{2}, \\
& n_{2}=n_{02}+\frac{\bar{n}^{6}}{4 \Delta n^{0}} g_{11}^{2}, \\
& n_{3}=n_{03}-\frac{\bar{n}^{6}}{4 \Delta n^{0}}\left(g_{11}^{2}-h_{3}^{2}\right) .
\end{aligned}
$$

Therefore the birefringence occurring for the light propagation along the $X$ axis is given by the relation

$$
\Delta n_{23}=n_{2}-n_{3}=\Delta n_{23}^{0}+\frac{\bar{n}^{6}}{2 \Delta n^{0}}\left(g_{11}^{2}-\frac{1}{2} h_{3}^{2}\right) .
$$

Following from Eqs. (14) and (16), one can derive the ellipticity of the eigen waves polarised in the $Z X$ plane as $\frac{D_{1}}{D_{3}}=i \kappa$ for the case when the linearly polarised incident electromagnetic vibration is parallel to the $X$ or $Z$ axis:

$$
\begin{aligned}
& \frac{D_{3}^{(1)}}{D_{1}^{(1)}}=i \kappa=-i \frac{\bar{n}^{3} h_{3}}{2 \Delta n^{0}}, \quad \kappa=-\frac{\bar{n}^{3} h_{3}}{2 \Delta n^{0}}, \\
& \frac{D_{3}^{(3)}}{D_{1}^{(3)}}=i \kappa=i \frac{2 \Delta n^{0} h_{3}}{\bar{n}^{3}\left(g_{11}^{2}-h_{3}^{2}\right)}, \quad \kappa=\frac{2 \Delta n^{0} h_{3}}{\bar{n}^{3}\left(g_{11}^{2}-h_{3}^{2}\right)} .
\end{aligned}
$$

It is worthwhile that Eqs. (19) may be simplified to the form given by Eq. (6) if only $g_{11}=0$. Moreover, the ellipticity is equal to zero under the condition $h_{3}=0$.

Using Eqs. (9) and (16), one can determine the ellipticity of the eigen waves for the other case of linearly polarised incident vibrations parallel to the $Y$ or $Z$ axis (i.e., those located in the $Z Y$ plane):

$$
\begin{aligned}
& \frac{D_{3}^{(2)}}{D_{2}^{(2)}}=i \kappa=i \frac{\bar{n}^{3} g_{11}}{2 \Delta n^{0}}, \quad \kappa=\frac{\bar{n}^{3} g_{11}}{2 \Delta n^{0}}, \\
& \frac{D_{2}^{(3)}}{D_{3}^{(3)}}=-i \kappa=-i \frac{\bar{n}^{3}\left(g_{11}^{2}-h_{3}^{2}\right)}{2 \Delta n^{0} g_{11}}, \quad \kappa=-\frac{\bar{n}^{3}\left(g_{11}^{2}-h_{3}^{2}\right)}{2 \Delta n^{0} g_{11}} .
\end{aligned}
$$

As one can see from Eqs. (20), simultaneous existence of the ordinary gyration and the weak optical activity leads to a difference in the ellipticities of the eigen waves with the $Z$ and $Y$ polarisations (the light propagates along the $X$ direction). Namely, the ellipticity of the wave with the polarisation parallel to the $Z$ axis depends on both the ordinary gyration and the weak optical activity, while that of the wave polarised along the $Y$ axis is a function of the ordinary gyration only. Notice that the absolute magnitudes of the ellipticities of the eigen waves are the same if the ordinary gyration is only present (see Eqs. (12)).

The longitudinal component $D_{1}$ in our case remains the same as in the case when the weak optical activity is only present (cf. Eqs. (6) and (19)). Thus, the coexistence of the 
gyration and the weak optical activity transforms the initial linearly polarised wave with the polarisation direction parallel to the $Z$ axis into the wave whose polarisation is of a 'mixed' longitudinal-transverse elliptical type (see Fig. 1). As far as we know, this represents a novel type of the eigen waves in crystals. The ratio of the displacement vector components is defined as:

$$
\frac{D_{2}^{(3)}}{D_{1}^{(3)}} \approx \frac{g_{11}}{h_{3}} .
$$

As seen from Eq. (21), traditional polarimetric methods (see, e.g., [9]) for the studies of ellipticities of emergent waves can be employed in order to reveal the weak optical activity. For instance, this may be accomplished after comparison of the ellipticities of waves initially polarised parallel to the $Z$ and $Y$ axes.

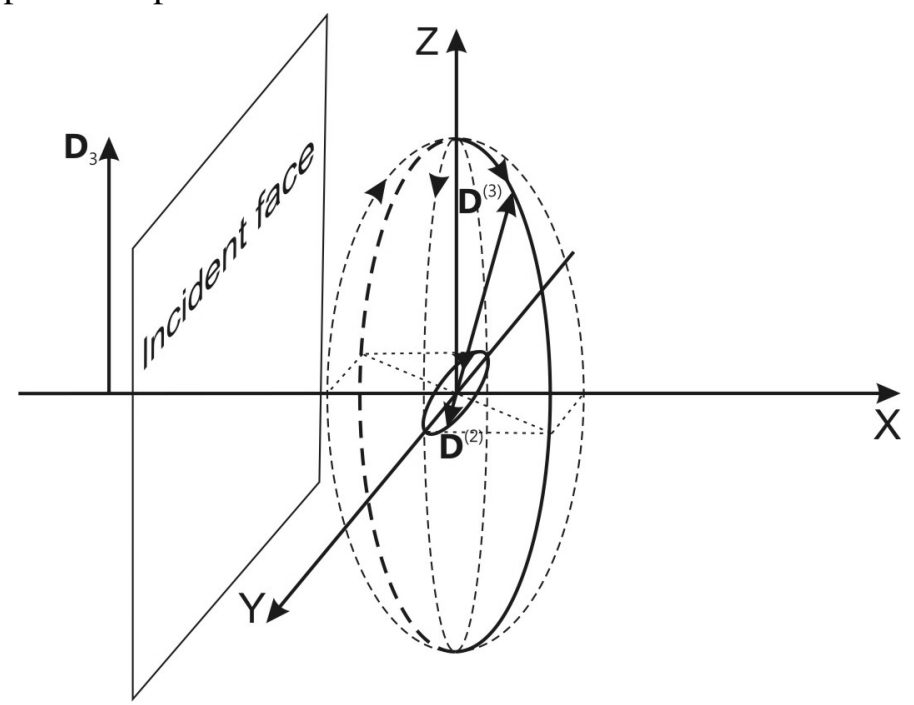

Fig. 1. Polarisation of eigen waves under conditions when the ordinary gyration and the weak optical activity are superimposed.

\section{Conclusions}

As a result of the present study, the relations describing the components of the impermeability tensor, the refractive indices, the birefringence, and the ellipticities of the eigen waves have been derived for the general case of superimposing ordinary gyration and weak optical activity. Basing upon our analysis of the ellipticity of polarisation and the refractive indices of the eigen waves characteristic of the crystals with coexisting gyration and weak optical activity, one can draw a number of important conclusions. The first is that the ellipticities of the eigen waves become different. Since this difference appears owing to the presence of the weak optical activity, one can suggest a purely polarimetric technique for revealing this phenomenon.

The second conclusion is that the polarisation state of one of the eigen waves (that of which major axis of the polarisation ellipse coincides with the direction of the constitutive vector of the weak optical activity) acquires a rather complicated form. It represents a 
longitudinally-transverse elliptical polarisation. This novel eigen state of polarisation comprises the two elliptical states, one of which is transversely elliptical and the other longitudinally elliptical.

\section{References}

1. Fedorov F I, 1959. On the theory of optical activity of crystals. 2. Crystals of cubic system and polar classes of medium-symmetry systems. Opt. Spektr. 6: 377-383.

2. Ivchenko E I, Permogorov S A and Selkin A V, 1978. Reflection of light with a change of polarization state from the real crystal boundary. Sol. St. Commun. 28: 345-348.

3. Ivchenko E L, Pevtsov A B and Sel'kin A V, 1981. Effects of optical activity on exciton luminescence of CdS. Sol. St. Commun. 39: 453-455.

4. Zil'bershtein A Kh and Solov'ev LE, 1998. Reflection of light with a change of polarization state from real crystal boundary. Opt. Spectr. 84: 549-552.

5. Lalov I J and Kojouharova N A, 2000. Reflection of electromagnetic waves at the boundary of optically active media. Bulgarian J. Phys. 27: 66-71.

6. Agranovich V M and Ginzburg V L. Crystal optics with spatial dispersion, and excitons ( $2^{\text {nd }}$ edition). Moscow: Nauka (1979).

7. Zapeka B, Kostyrko M and Vlokh R, 2010. On the light refraction and polarisation in the presence of weak optical activity. Ukr. J. Phys. Opt. 11: 68-73.

8. Sirotin Yu I and Shaskolskaya M P. Fundamentals of crystal physics. Moscow: Nauka (1979).

9. Vlokh O G. Spatial dispersion phenomena in parametric crystal optics. Lviv: Vyshcha Shkola (1984).

Zapeka B., Kostyrko M. and Vlokh R., 2010. Coexistence of ordinary gyration and weak optical activity in crystals: eigen waves of a new type. Ukr.J.Phys.Opt. 11: 119-125.

Анотація. У роботі представлено результати аналізу показників заломлення $і$ еліптичності власних хвиль за умови співіснуванні гірачії і слабкої оптичної активності. Отримано кількісні співвідношення, щчо описують дані параметри. Показано, щчо еліптичності власних хвиль відрізняються. Встановлено, щзо одна з власних хвиль набуває нового, складного поляризачійного стану - так званої поздовжньо-поперечної еліптичної поляризації. 